\title{
Analysing the hindrance to the use of information and technology for improving efficiency of health care delivery system in Tanzania
}

\author{
H.C. KIMARO* \& H.M. TWAAKYONDO \\ University of Dar es Salaam, Department of Computer Science, Dar es Salaam, Tanzania
}

\begin{abstract}
Information Technologies (IT) have been described as offering tremendous opportunity to improve health services as well as in meeting broader developmental goals which have an impact on health. Through the use of IT, healthcare sectors can potentially plan, monitor and evaluate health services as well as communicate more effectively within and across organizational hierarchies. However, a number of studies suggest several hindrances where the use of IT to bring critical change in the health sector of Tanzania has been problematic. Despite the lack of appropriate use of the existing IT resources in the health sector, donors and government have continued helping the health sector to acquire up-to-date IT resources while however placing little emphasis on long term IT training, data management and effective utilization of information resulting into wasted of such resources hence little improvement in health services delivery. This study is based on the Health Information System Programme (HISP), an action research project aimed at improving health information system in developing countries with the use of IT and information for local action. Under the project, the district health information software which is customizable, open source and freely distributed has been implemented in five pilot districts in Tanzania. The lessons learned from HISP project and other levels of the health sector in general indicate the lack of skills for data interpretation and utilisation, policy guidelines on information and human capacity building as well as a lack of flexible system.
\end{abstract}

Key words: health, information technology, data management, Tanzania

\section{Introduction}

Developing countries have often been challenged to improve their Health Information Systems (HIS) through the use of information technology (IT) in order to upgrade the health status of their people (World Bank, 1994; Braa \& Blobel, 2003). The Tanzanian health sector in particular responded to this call by computerising its HIS at the regional and national levels. One of the aims is to develop an IT based HIS that is reliable, integrated and functional. However, despite tremendous local and international efforts and resources spent during the last decade on the HIS development and implementation, the system is still unreliable due to many factors including a lack of culture of information and IT use, appropriate human capacity, infrastructure, and administrative commitment as well as a lack of flexible system design. In addition, these factors have led to lack of timely, reliable and relevant data/information as well as use of information for action (Mwangu, 2003; Wambura 1998; Mwangu \& Otito, 2000).

The Health Information Systems Programme (HISP) was established in South Africa in 1994 and is today the official health information system in the country (Braa \& Hedberg 2002). Its relative success in South Africa has led to the adoption of the software and ideas on health management to countries such as Mozambique, India, Malawi, Mongolia and Cuba (Braa et al., 2004).

The HISP focuses on action research, user participation, especially local participation to adapt the District Health Information Software (DHIS) with the aim of developing local knowledge and skills in computers, data handling and use (Braa et al., 2004, Williamson et al., 2001). Ever since it was developed in 1997, DHIS has gone through many improvements that are applicable to all countries, but it is also adapted in every country to meet the needs of the local contexts (Braa et al., 2004). Since 2002, the Tanzanian HISP team has implemented the HISP in five pilot districts. This paper seeks to address the status of current Tanzanian HIS and identify major hindrances to the effective and long term use of information and technologies in the health sector.

Most Health Information Systems in developing countries are designed to follow data-led approach where massive data is collected but with limited use rather than the action-led approach where the focus is on information that supports management decisions and emphasis on data collectors as the main information users (WHO, 1994; Lippeveld et al., 1997; Heywood \& Rohde,

\footnotetext{
* Correspondence: Honest C. Kimaro; Email: honestck@ifi.uio.no
} 
2000). The data handling is carried out to reflect the need of the higher level bureaucracy rather than information needs for local actions (Sandiford et al., 1992). The HIS does not become part of organisational routines because of lack of ownership, insufficient support and low capability of health workers at local levels. The HIS design is also not made flexible to evolve along with dynamic institutional and technological changes (Bisbal et al., 1999) which in turn require constant availability of financial resources to cope with new changes across time and space, something most developing countries cannot afford in practice.

The aim of this study was to apply action research to provide health workers and researchers with a chance to work together to effectively design and operate IT and use information for action, share learning and experiences and to become more aware of the options and possibilities for changing in their context. HISP uses the District Health Information Software (DHIS) (Braa \& Hedberg, 2002) to assist in the process of storing, analyzing and presenting routine health data for making informed decisions. The key intervention strategies are trainings, workshops and participatory design (Walsham 2002; Greenbaum \& Kying, 1991) of the DHIS to facilitate learning and use.

\section{Materials and Methods}

The HISP project was initiated in the Bagamoyo and Kibaha districts in Coast region in 2002. Bagamoyo $\left(6^{0} 15^{\prime} \mathrm{S}, 33^{0} 58^{\prime} \mathrm{E}\right)$ is located about $70 \mathrm{~km}$ north of the city of Dar es Salaam with an area of $9,842 \mathrm{~km}^{2}$ and a population of about 230,164 people with some 26 public health facilities. Kibaha district $\left(6^{0} 50^{\prime} \mathrm{S}\right.$, $\left.38^{0} 50^{\prime} \mathrm{E}\right)$ is located about $40 \mathrm{~km}$ west of Dar es Salaam with a population of about 132,045 and some 19 public health facilities.

The study was based on a number of interviews with health workers at various health facilities, health information compilers, secretaries and managers as well as participant observations. Group discussions, meetings, workshops and training sessions were conducted in different periods between 2002 and 2005 at the Ministry of Health headquarters, the Coast Region health office, and the Bagamoyo and Kibaha districts. In addition, analysis was performed on documents such as data forms and other unpublished reports. The analysis of the empirical data was carried out based on the interpretive and participatory action research framework. The "interpretive" research approach assumes that knowledge to be context-bound and dependent on what actors subjectively do in particular situations. It aims at the analysis and description of human behaviour by an understanding of the meanings individuals attach to their actions (Walsham, 1993).

The two districts, Bagamoyo and Kibaha were earmarked as initial HISP pilot sites. At very early stage in July 2002, HISP team in collaboration with the head of the HMIS unit at the Ministry of Health headquarters using sample data from Bagamoyo district organized a presentation of the HISP database tool, namely, the DHIS at the Ministry of Health headquarters. The aim of this presentation was to include more stakeholders in the HISP and to build awareness of the project from the national level. In the following year, 2003, HISP team further organized intensive user trainings of the DHIS tool along with the study of data flows and use in both pilot districts. In 2004, while providing user support in the two pilot sites, the scaling and training of the DHIS was undertaken in three other districts namely Ilala, Temeke and Kinondoni in Dar es Salaam.

\section{Results}

\section{Data handling processes}

Generally, despite the large collection of Health Management Information System (HMIS) registers, forms, and tally sheets, some datasets were identified as missing, leading to some curious health workers to invent local tools to record their data resulting to data inconsistent and contradictive interpretations at the higher levels. When asked about missing data elements in the HMIS registers, one of the national technical managers replied:

When the changes are introduced, it means a need to train individuals on those changes nationwide, which may take a long time and lots of financial and technical resources. It took more than two years for some individuals to understand how the current data register books and procedures work. Even though currently 
there are some regions, which have not been able to utilize them properly.

Moreover, sometimes the register books for data collection are not distributed to the health facilities on time from the higher levels. For example, in 2002, one of the health districts received the books seven months late. During one of the researchers' visits in February 2004 the one of the two districts had not received the books. Thus most of the health facilities visited were either using the previous year's register books or using their locally structured forms, trying to improvise them as register books. It was noted that these registers were procured and distributed behind schedule because of the lack of funding to support the printing and distribution processes.

There was no indication of feedback from higher levels to lower levels as was noticed even though the health officials at the national level (Ministry of Health) said that they did provide an overall annual report with a summary of all the regions in Tanzania. However, in contrast to what was said at the Ministry, at the health facility level, health workers spoke of the feedback they sometimes get only in terms of reports they had sent being returned back for corrections such as missing data. However one of the health facility in-charge said that even when feedback is provided, authorities might fail to implement because usually health facilities do not get any funding from the government. This also weakens the process of data handling and use.

Despite the fact that the district Council Health Management Team (CHMT) is composed of District Medical Officer (DMO), District Health Officer (DHO), District Nursing Officer (DNO), District Laboratory Technologist, District Dental Officer, District Pharmacist, District Health Secretary, District Health Education as well as well as Programme Coordinators/Focal persons for Malaria, Tuberculosis/Leprosy, HIV/AIDS, Reproductive and Child Health, data management was the responsible of the HMIS focal person. When one of the programme coordinators at the regional level was approached regarding data processing at the district he responded:

It could be better that a person dealing with MCH for example to cooperate with HMIS coordinator. The only person dealing with data is HMIS coordinator. This system is not for a single person to deal with. Now the vertical programmes have gained momentum in data collection since they do not get reliable information through HMIS".

Thus, the HMIS reporting system and a number of surveillance systems impose a high work burden on information compilers at the health facilities.

\section{Human resource capacity}

During the introduction of HMIS training was provided to health facility workers on how to collect data and/or health indicators using the new tools. The last training had focused on mistakes which the health workers had made in filling the registers. A three-day training was also given to some members of the CHMT on how to process data reported from health facilities and how to calculate health indicators. To-date most health workers responsible for data collection and reporting were not trained on how to use the HMIS system. One of the district health secretaries pointed out that, there is a need for ensuring that data collectors are empowered by building their capacity. But, "the problem is that most of the time the data collectors are busy with issues of direct health services."

For the health facilities attending to more patients than their service area, it becomes difficult for one health worker to compile the report, since the same health worker attends to many patients. Health workers at the facility level are responsible for data collection from the community, i.e., visiting the villages but this is not always done due to lack of resources as one of the District HMIS coordinator expressed it: "...we have not used the community book for so long... because health workers at the facility are few... have no time to do that...there are no funds for visiting the community."

Until recently, the districts directly reported computer faults to the national level but most issues had been delegated to the respective level depending on their financial capability to hire someone from a private company for computer service and maintenance. However this approach received criticism for paying a lot of money for solving simple technical problems such as fixing cables and installing software. When asked about employing a permanent person for helping with technical 
problems, the Regional Health Administrative Secretary had these to say:

Currently the Ministry of Health want to have a manageable size of staff. In order to introduce a new professional such as IT that does not exist within the Ministry of Health, the department of civil services must approve it. Otherwise later on we will not be able to promote him.

\section{Supervision of data handling processes}

Health facilities are to be supervised by the CHMT who are supposed to visit each and every health facility in their respective district every month. The supervision process involves checking every HMIS register to see that data are correctly recorded and consistent with reports prepared. Supervision visits are also regarded as a way for the CHMT to provide feedback to health facility in-charge and to provide other guidance.

In one of the districts, the HMIS coordinator said that when he visited the health facilities in February 2004, out of 37 facilities he managed to visit 22 facilities to collect the reports. Since the supervision goes in parallel with collecting and validating the quarterly reports from the health facilities, this is the time when he was collecting reports for December 2003. However, he expressed that it was hard for one person to visit a large number of health facilities per day while collecting and validating the reports. Thus, data validation at the facility level lacked supervision from the CHMT.

\section{Decision making processes at various levels}

There was no evidence found regarding the use of HMIS information other than health workers using information to know how many and what medicines are being used, the services that they are providing, the use of equipment and what additional equipment are required. This indicated that most of the health workers fail to understand the purpose of collecting data, as it is expressed in the following statement by one of the District Health Secreatry:

Most health workers do not understand the purpose of health data collection, resulting in poor reporting.

The limitations and constraints on information, human capacity and IT at lower levels (facility, district and regional) are summarised in Table 1. There was a general assumption that data is only needed at the Ministry of Health where planning and decision making was to be done for the lower levels. Thus, the Ministry of Health had the information, power and authority to tell the lower levels what to do in order to improve their health status. Another problem expressed was how the lower levels work under orders from the higher levels as well as from donors.

We tell the district that we need this kind of report and then they make that report for us. That is the kind behaviour we have created in our society (Regional Programme Coordinator) He went on to say that:

There is no initiative to use data at lower levels. The national level is forced by the Wolrd Health Organization. They do that because somebody is behind them and they have money. If they are late, they receive a telephone call to report the data.

However, most of the information generated through the HMIS was inconsistent with what the management at the lower levels needed for decision making as the lower levels were not involved in the system design process.

\section{Impacts of HMIS on HISP}

Since the data generated through the HMIS are incomplete, the information generated from the DHIS application is equally regarded as incomplete. Thus, this problem of data quality continues to make the DHIS unable to generate the required benefits without deliberate efforts to create a culture of information dependence and use that will help to improve the data handling processes.

However, the DHIS is used along with these efforts to help in the analysis of data. The HISP team has been involved in training of health workers and trainers, continuous user support and participation in active discussions with health officials at various levels of the Ministry of Health. However, several factors have hindered HISP efforts including lack of active participation of health officials in the trainings and negotiations, lack of authority and mandate to make changes and lack of local skills and time for practice coupled with unavailability of proper authority when needed as well as the strong influences of the installed base (HMIS). Below, we describe different strategies adopted by the HISP 
Table 1: Limitations and constraints on information and IT use and human capacity in Kibaha and Bagamoyo districts

\begin{tabular}{|c|c|c|}
\hline HMIS & $\begin{array}{l}\text { Limitation and } \\
\text { constraints }\end{array}$ & Causes \\
\hline \multirow[t]{4}{*}{$\begin{array}{l}\text { (1) } \\
\text { Data/informat } \\
\text { ion }\end{array}$} & Lack of timeliness & $\begin{array}{l}\text { Lack of resources (materials, human, } \\
\text { financial) } \\
\text { Lack of skills and motivation } \\
\text { Heavy workload (lack of time) }\end{array}$ \\
\hline & Poor quality data & $\begin{array}{l}\text { Missing/incomplete data } \\
\text { Large data sets } \\
\text { Inaccurate data } \\
\text { Lack of data validation or verification } \\
\text { (supervision) }\end{array}$ \\
\hline & Lack of data analyses & $\begin{array}{l}\text { Lack of skilled personnel } \\
\text { Lack of proper data analysis tools } \\
\text { Lack of use of information }\end{array}$ \\
\hline & $\begin{array}{l}\text { Data not used in decision } \\
\text { making }\end{array}$ & $\begin{array}{l}\text { Lack of skills to use data } \\
\text { Lack power of making decisions } \\
\text { Culture that emphasizes on collecting and } \\
\text { reporting information }\end{array}$ \\
\hline \multirow{2}{*}{$\begin{array}{l}\text { (2)Personnel: } \\
\text { Health } \\
\text { Workers/Heal } \\
\text { th managers }\end{array}$} & $\begin{array}{l}\text { Lack of skills, knowledge and } \\
\text { practice }\end{array}$ & $\begin{array}{l}\text { Lack of appropriate training on data } \\
\text { handling, analyses and interpretation }\end{array}$ \\
\hline & $\begin{array}{lr}\text { Lack of } & \text { motivation, } \\
\text { ownership, } & \text { and } \\
\text { empowerment } & \end{array}$ & $\begin{array}{l}\text { Low salary and lack of incentives } \\
\text { Heavy work load } \\
\text { Poor working environment }\end{array}$ \\
\hline \multirow[t]{3}{*}{$\begin{array}{l}\text { (3) IT/HIS } \\
\text { software }\end{array}$} & Software design limitations & $\begin{array}{l}\text { Unmodifiable data fields (hard coded) } \\
\text { Absence of source code, (lack of ownership of } \\
\text { the source code) and prompt user support } \\
\text { mechanism } \\
\text { Lack of data validation mechanism and other } \\
\text { standard functionalities }\end{array}$ \\
\hline & $\begin{array}{l}\text { Inadequate technical } \\
\text { knowledge and resources }\end{array}$ & $\begin{array}{l}\text { Short term training e.g. MS Word and some } \\
\text { EXCEL }\end{array}$ \\
\hline & $\begin{array}{l}\text { Lack of hardware } \\
\text { maintenance } \\
\text { Lack of data management } \\
\text { utility such as data backup } \\
\text { facilities } \\
\text { Lack of update Anti-Virus } \\
\text { Software and other IT security } \\
\text { mechanisms }\end{array}$ & $\begin{array}{l}\text { Lack of practice and participation in design } \\
\text { Lack of long term policies on IT cadre and } \\
\text { advanced IT training }\end{array}$ \\
\hline
\end{tabular}

team to cultivate an information use culture despite the identified barriers.

\section{Strategies adopted by HISP}

The HISP team has been engaged in organizing different on/ off site training for data collectors and health managers at the district level with invitation of other higher levels. The trainings involved basic computer use, the DHIS, and data handling skills. In parallel with training, the HISP team has been analyzing the routine health data from the health facilities using the DHIS to generate reports identifying gaps on data for the management action. Moreover, some HISP members have also been involved in individual research, studying data handling processes, flows, and decision making in the multiple levels of the Ministry of Health that have contributed significant inputs to the HISP and the Ministry of Health (Lungo, 2003; Mwangu, 2003). 


\section{Challenges encountered by HISP}

There has been a lack of commitment by the district health authorities/health officials to participate in the HISP training programmes coupled with lack of authority to deal with the reported data handling problems. Likewise, supervision in most cases was not done to provide health workers with appropriate learning on data processing and use. HISP trainings conducted had never involved district authorities, apart from HMIS coordinators and other health workers as they have never had time. Moreover, the district level depended on higher levels on many decisions. For example, it was almost impossible for district authorities to decide to change the HMIS reporting to the district from quarterly aggregated data ('HMIS book 10') reporting to monthly aggregated data ('HMIS book 2') reporting as was suggested by the HISP in order to make it easier to trace poor quality data.

A strong political resistance existed from the existing HMIS regarding costs and resources incurred even though no positive results have been achieved. However, the Ministry of Health officials said they are ready to give support to HISP, while running the DHIS and HMIS database in parallel until when the policy makers are convinced of the HISP potential over the HMIS.

...we do not want to move quickly this time with something that we have not realized its output,...the policy makers need to be convinced of the HISP/DHIS,... (Head, HMIS Unit)

However, the local trainees perceived the DHIS as just a tool to store data (data repository tool) rather than to generate usable outputs because the district authorities never asked for processed information for decision making. For example, since the DHIS was installed in the two initial pilot districts, health workers have just enjoyed putting data into the DHIS. This is however because the data compilers can only process data while the health managers have the task of using that information for decision making. However, managers did not participate in knowing what the DHIS is all about. The health workers (data compilers) being involved in direct health services (curative services) and other duties occasionally get time to use the DHIS thus tending to forget how to operate some of the functions as it was expressed by the HMIS coordinator in one of the pilot districts.

...we are not used yet for decision making. All the data entered are needed. The knowledge to generate a general report is yet clear. Also I do not have enough time to settle down and sit at the computer. I need to learn more.

Thus, the use of the DHIS seemed to base on personal motivation to learn the use of computer and not for organizational purposes. With most health facilities understaffed and some located in difficult to reach places due to poor transportation and roads (for example in Bagamoyo district) and a general lack of financial capacity and institutional support this has made it difficult to organize training of health workers at facility level.

Moreever, the HISP team faced some difficulties in defining exactly the information needs of the district organization due to lack of capability and power of the district level that depends on the national level coupled with lack of practical participation of district health management team members who were busy with other managerial activities and direct health services.

\section{Discussion}

Our findings suggest that the lower the level, the lower the understanding of the meaning of data collected. The health workers at health facilities are the least capable in understanding of the use of information though they are the producers of that very information. We argue for a more interactive structure which allows learning, collaboration and sharing of knowledge to balance the general understanding of the meanings and values of data. There was limited evidence in terms of values of information or resources to be drawn upon by health workers in the data handling processes because for them data is for reporting and showing how they used the resources they are getting from higher levels. The health workers had no enough knowledge, skills and resources to make sense of information and they lacked an understanding of the general role of the HMIS in their work of providing direct heath (curative) services. In general, the design and implementation of HMIS was undertaken without strategies to develop adequate knowledge and long term training in the area of HIS, 
IT and use of information for decision making (Mwangu, 2003; Miller et al., 2003; Kagonji, 2005).

At the health facility which is the source of all routine data there is no evidence of use of data, routine feedback or regular training. This leads to a perception that reporting is to be done for the sake of the system, and consequently it is perceived as secondary to the provision of direct health services. A consequence of this is little emphasis on collecting accurate data, as well as late or missing reports.

The supervision is not to be regarded as a way of fixing identified mistakes on the data but rather to provide guidance and learning as well as to provide mutual understanding on the importance of data. Each level is given the responsibility to supervise its immediate level below, for example, health facilities are to be supervised by the CHMT who are supposed to visit each and every health facility in their respective district. However, only few health facilities were able to report that the supervision had been done but in terms of criticizing poor handling of data. Similar findings have been reported from a study carried out in Dodoma and Babati districts in Tanzania in 2002 (Miller et al., 2003).

The lack of supervision and training has led to the lack of appropriate meanings of information collected coupled with lack of skills. We found the existing data anomalies by inputting all the data on the DHIS to produce various reports such as health facilities which did not report as well as data that have not been reported by particular facilities as a way of disclosing the problems. However, the culture emphasized data collection and reporting regardless of whether data was used locally or not. Health workers in the health facilities had to use any means to prepare the reports (regardless of data quality) even with lack of time and with others using rough papers for collecting data, making information even harder to make sense of. In two districts visited, the CHMT members had no formal collaboration mechanism to analyse, aggregate or discuss data handling problems among themselves or with vertical programmes coordinators so as to find a way to deal with those issues in a collaborative manner.

The interventions led by the HISP team to train the health workers on the meaning of information, means to capture data and using computers and the DHIS at the district level is an attempt at shaping the existing culture of collecting, reporting through use of information to create a more modest information use culture. However, this requires an institutional and managerial support along with financial resources from the national level. Indeed, the national level cannot just stay, see and wait for the HISP to achieve progress without their participation in the process as this does not motivate the lower levels and as the lower levels work to satisfy the national level's interest.

The health managers at all levels of the heath sector need to show in practical actions that the information/data collected is needed and relied upon for the management of health care services. Currently, the lower level health managers and health workers follow orders from higher level to act on data at their locality which contradicts with the general essence of action-led approach (Lippeveld et al., 1997; Heywood \& Rohde, 2000). The set of norms and resources must be enhanced for each individual level and are subject to be changed to create a culture of information use. Indeed, the interdependence, interaction and integration that exist between levels can be used to enhance learning through proper supervision along with participation in joint local actions. Thus, local changes must be appreciated/supported by or go along with changes at the higher levels thus calling for a participative bottom-up and top-down approach for interventions at local levels. The need to address issues at multiple levels is important (simultaneous top-down and bottom-up) to secure both institutional mandate, participation and ownership.

People within the HMIS are part of the installed base. Thus, development of a new HIS must go along with development of appropriate human resources so as to create new capability and skills. Thus, allocation of sufficient resources and appropriate strategies are needed to support long term training (on/off site) as long as people and heath care institutions need new skills and knowledge in work processes, interpretation of information and IT. In-house training for capacity building is possible with some financial capacity and IT resources available at various levels of the health system. However, the lack of policy 
guidelines on information use and IT makes it difficult for health managers to put emphasis on the use of information as well as to deal with human resource issues for IT use and support.

The flexible, open source and customisable software system unlike rigid systems like HMIS software will help when the manual system is changed following new organisational needs thus avoiding starting a new software development project. For example in 1997, the HMIS paper based system's data reporting frequency was changed from monthly to quarterly along with introduction of new forms. These changes led to the start of a new project to develop new software in MS Access. Thus, when unpredictable organisational change occurs, it has not been possible to adapt the HMIS system to these changes. The hard coding of data elements makes the HMIS software inflexible. However, since the source code is also not available, the software can not be changed by the Ministry of Health alone without a paid support of the developer. Also the availability of funding to pay the developer to deal with the changes often depends on donors.

The HIS being regarded an information infrastructure (Hanseth, 2002; Hanseth \& Monteiro, 1996), its design and development must take into account existing cultures and skills as well as work procedures of data collection, analysis and use and the needs of available vertical programmes. The information infrastructure perspective also demands that the system such as HMIS could not be designed or developed out of its operational and cultural context. Thus, development of HIS need to include a critical study of installed base and its operational and cultural context and need to be carried out in a participative way involving all levels in the process. Since the installed base is deeply embedded in the existing organisation culture, it constitutes a barrier towards change as problems often are not just technical but also non-technical. Thus, the installed base can be used as a resource for building a new HIS that is reliable, functional, and flexible.

The HIS and health sector in general require several institutional and cultural changes to cultivate appropriate attitudes towards information and IT use. We thus argue for decentralization of authority (Walsham, 1992), improving skills on interpretation of data and use of IT along with changes in work procedures of HIS. The deliberate efforts to enhance collaboration among levels along with changes in ideas, ways of thinking about information and using information are also needed. However, these changes cannot be carried out overnight or imposed from top levels but requires managerial commitment at all levels and must be carried out in an incremental manner while involving accumulation of resources and participation of all levels (and other stakeholders) to gradually change peoples' deeply embedded attitudes and behaviours toward information use. The continuous training and learning environment is necessary to create new meanings, techniques and work procedures of data processing and use and thus evolve a new culture of information use.

Both participative bottom-up and top-down HIS design approaches (Mwangu, 2003) are required to create a sense of ownership of the HIS at all levels as well as to help to build culture and skills that give importance to information use for local action. By learning through past problems, and through regular training, people can attain appropriate skills whereas through participative processes, peripheral workers can be motivated and given needed confidence on what they do. However, both political and managerial commitments are needed to create appropriate information use and IT policies, working environment and procedures, and human capacity development strategy as well as allocating timely resources. Moreover, the HIS is to be regarded as an information infrastructure and thus making its design as flexible as possible to allow smooth extension of its design to accommodate new needs and cultural values over time.

\section{References}

Bisbal, J., Lawless, D., Wu, B. \& Grimson, J. (1999) Legacy of information systems: Issues and directions, IEEE Software, September/October 1999. pp. 103-111.

Braa, J. \& Blobel, B. (2003) Strategies for Developing Health Information Systems in Developing Countries. In: WITFOR 2003 White Book. D. Khakhar. Luxenburg, IFIP Press: pp.175-219. 
Braa, J. \& Hedberg, C. (2002) The struggle for district-based health information systems in South Africa. The Information Society 18, 113127

Braa, J., Monteiro, E. \& Sahay, S. (2004) Networks of action: sustainable health information systems across developing countries. MIS Quarterly 28, 337-362.

Greenbaum, J., \& Kying, M. (1991) Introduction: Situated Design. In Design at work: Cooperate Design of Computer Systems. J. Greenbaum \& M. Kyng (eds), Lawrence Erlbaum Associates. pp. 1-24.

Hanseth, O. \& Monteiro, E. (1996) Information infrastructure development: the tension between standardization and flexibility. Science, Technology \&Human Values 21, 407-426.

Heywood, A. \& Rohde, J. (2000) Using information for action. A manual for health workers at facility level. The Equity Project, Pretoria, South Africa.

Kagonji, I.S. (2005) Knowledge, attitude and practice of health workers towards the use of health management information system in Iringa Municipality-Tanzania. Tanzania Health Research Bulletin 7, 43-45.

Lippeveld T, Saueborn R, Sapirie S. (1997). Health information systems: Making them Work. World Health Forum 18 (2), pp. 176-184.

Lungo, J. H. (2003). Data Flows in Health Information Systems: An Action Research Study of Reporting Routine Health Delivery Services and Implementation of Computer Databases in Health Information Systems. Department of Informatics, Oslo, University of Oslo, website: ttp://www.ub.uib.no/elpub/NORAD/2003/uio/t hesis01.pdf. Accessed October 2003.

Miller, L.F., Fields, R., Mmbuji, P.K.L., Posner, S., Mboera, L.E.G., Jimmerson, A., Senkoro, K.P., Rumisha, S.F., Shayo, E.H. \& Mwami, J.A. (2003) Situation Analysis of Infectious Disease Surveillance in two Districts in Tanzania, 2002. Bethesda, MD: The Partners for Health Reformsplus Project, Abt Associates Inc.

Mwangu, M.A. (2003) Enhancing District Health Planning and Management in Tanzania: A social political analysis of the role of the Health Management Information System (HMIS). Doctoral Thesis, University of Dar es Salaam, Tanzania.

Mwangu, M.A. \& Otito, C.K. (2000). HMIS implementation at the district level in the context of the Health Sector Reforms: Challenges and future prospects. Proceedings of the $18^{\text {th }}$ Annual Scientific Conference of the Tanzania Public Health Association, November 22-25, 1999, Dodoma, Tanzania. Pp 111-119

Sandiford, P., Annet, H. \& Cibulskis, R., (1992) What can information systems do for primary health care? An international perspective. Social Science and Medicine 34, 1077-1087.

Walsham, G. (1992) Decentralization of Information Systems in Developing Countries: Power to the People?. Social Implications of Computers in Developing Countries. S.C. Bhatnagar \& M. Odedra-Straub. Delhi: Tata McGraw-Hill.

Walsham, G. (1993) Interpreting Information systems in Organizations, Wiley, Chichester.

Walsham, G., (2002). Cross-cultural software production and use: A structurational analysis, MIS Quarterly 26, 359-380.

Wambura, C.M.M. (1998), Evaluation of Health Management Information System in Tanzania. Advanced Diploma Thesis in Public Health, Institute of Development Management, Mzumbe, Tanzania

WHO (1994) Information Support for New Public Health Action at the District Level. Geneva.

Williamson, L., Stoops, N. \& Heywood, A. (2001). Developing a District Health Information System in South Africa: A Social Process or Technical Solution?, Vol. 84 of Studies in Health Technology and Informatics, medinfo 2001 edn, IOS Press, Amsterdam, Chapter 8. pp. 773-777.

World Bank (1994) Better Health in Africa: Experiences and Lessons learned. Development in Practice, World Bank 